\title{
ANÁLISE ESPAÇOTEMPORAL DA EVAPOTRANSPIRAÇÃO NA ÁREA DE PRESERVAÇÃO AMBIENTAL DA ILHA DE SANTA RITA, ALAGOAS, BRASIL ${ }^{1}$
}

\author{
Admilson Penha Pacheco², Michelle Adelino Cerqueira ${ }^{3}$, Bernardo Barbosa Silva ${ }^{4}$ e Rosilene Mendonça
} Nicácio ${ }^{5}$

\begin{abstract}
RESUMO - A evapotranspiração (ET) é uma das principais componentes do ciclo hidrológico. Nesse contexto, este trabalho teve como objetivo analisar o comportamento espaço-temporal do Balanço de Energia e da Evapotranspiração Real Diária na Área de Preservação Ambiental da Ilha de Santa Rita, Alagoas, Brasil, tomando como subsídio as possíveis alterações no uso e cobertura do solo. A metodologia utilizada envolve o uso do algoritmo SEBAL, imagens do satélite Landsat 5 - TM e dados complementares de estação meteorológica localizada na área de estudo. A estimativa diária de $\mathrm{ET}_{24 \mathrm{~h}}$ real variou de 0 a $1 \mathrm{~mm} \mathrm{dia}{ }^{-1}$ no solo exposto e na cidade; a $6 \mathrm{~mm} \mathrm{dia}^{-1}$, em corpos d'água e mangue, com um valor médio de 3,78 $\mathrm{mm} \mathrm{dia}^{-1}$, 3,45 $\mathrm{mm} \mathrm{dia}^{-1}$ e 4,75 $\mathrm{mm} \mathrm{dia}^{-1}$, nos dias 03/09/2003, 26/08/2006 e 17/03/2011, respectivamente, em toda a área estudada. Os resultados por meio do algoritmo SEBAL (SURFACE ENERGY BALANCE ALGORITHMS FOR LAND) podem ser utilizados em condições climáticas semelhantes às da Área de Preservação Ilha de Santa Rita/Alagoas. As distribuições de cada componente do balanço de energia: saldo de radiação, fluxo de calor no solo, sensível e latente, apresentaram influência significativa nos diferentes tipos de uso e cobertura do solo. Além disso, constataram-se diferenças evidentes entre os fluxos em zona rural e área urbana. A abordagem utilizada é muito adequada para uma exploração real de dados de satélite, podendo ser útil para estimar um número de parâmetros no contínuo solo-planta-atmosfera.
\end{abstract}

Palavras-chave: Sebal; Balanço de energia; Evapotranspiração.

\section{SPACE-TIME ANALYSIS OF EVAPOTRANSPIRATION IN THE ENVIRONMENTAL PRESERVATION AREA OF ILHA DE SANTA RITA, ALAGOAS, BRAZIL}

\begin{abstract}
The evapotranspiration (ET) is one of the most important components of the water cycle. On this aspect, this study aims to analyze the space-time behavior of the energy balance and the real daily evapotranspiration in the area of environmental preservation of Ilha de Santa Rita, Alagoas, Brazil. The methodology used involves the use of algorithm SEBAL, satellite images of Landsat 5 TM and complementary data of meteorological station located in the study area. The daily estimate of real $E T_{24 h}$ varied from 0 to $1 \mathrm{~mm}^{-d a y^{-1}}$ for exposed soil and city; to $6 \mathrm{~mm}$.day ${ }^{-1}$ for water bodies and mangrove, with a mean value of $3.78 \mathrm{~mm}$.day-1 $3.45 \mathrm{~mm}^{- \text {day }^{-1}}$ and 4.75mm.day-1, in the days 09/03/2003, 08/26/2006 and 03/17/2011, respectively, for the entire study area. The results obtained by the algorithm SEBAL (SURFACE ENERGY BALANCE ALGORITHMS FOR LAND) can be used in weather conditions that are similar to the ones in the preservation area of Ilha de Santa Rita/ Alagoas. The distributions of each component of the energy balance are: radiation balance, soil heat flow
\end{abstract}

\footnotetext{
${ }^{1}$ Recebido em 19.06.2013 aceito para publicação em 14.04.2014.

${ }^{2}$ Departamento de Engenharia Cartográfica, Universidade Federal de Pernambuco, PE, Brasil. E-mail: <pacheco3p@gmail.com>.

${ }^{3}$ Pós-Graduanda em Ciências Geodésicas e Tecnologia da Geoinformação, Universidade Federal de Pernambuco, PE, Brasil. E-mail: <ac_mi@hotmail.com>.

${ }^{4}$ Departamento de Engenharia Civil, Universidade Federal de Pernambuco, PE, Brasil. E-mail: <bbdasilva.ufpe@gmail.com>.

${ }^{5}$ Departamento de Engenharia de Agrimensura da Universidade Federal de Alagoas, Brasil. E-mail: <rmnicacio@gmail.com>.
} 
(sensible and latent), presented significant influence in the different types of use and coverage of the soil. The approach used is very suitable for a real satellite data exploration, and it can be used to estimate the number of parameters in the continuous soil-plant-atmosphere.

Keywords: Sebal; Energy balance; Evapotranspiration.

\section{INTRODUÇÃO}

A evapotranspiração (ET) pode ser definida como a perda de água para atmosfera a partir do solo, lagoas, lagos e superfícies vegetativas devido à vaporização da água líquida (IRMAK et al., 2011), sendo definida pela soma da evaporação e transpiração, em que a combinação desses dois processos ocorre de forma separada.

Segundo Allen et al. (2002, 2011b), a evapotranspiração é variável no espaço e no tempo: variável no espaço devido à grande variabilidade espacial da precipitação, características físico-hídricas do solo e ao tipo e densidade da vegetação; e variável no tempo, em razão da variabilidade do clima. Portanto, a gestão eficiente dos recursos hídricos depende da correta estimativa da evapotranspiração e dos diferentes componentes na superfície da terra.

A evapotranspiração pode ser medida por meio de equipamentos, como os lisímetros, ou estimada por meio do balanço hídrico ou de dados meteorológicos aplicados em equações, como a utilizada pela FAO 56 Penman-Monteith (ALLEN et al., 1998). Porém, esses métodos são limitados porque eles estimam valores pontuais de ET para um local específico e não em escala regional. Essa limitação motivou o desenvolvimento do uso de dados obtidos por sensoriamento remoto, em que a principal vantagem da aplicação desse procedimento está baseada na evaporação, em que a água é consumida pelo sistema solo-água-vegetação e pode ser obtida diretamente sem a necessidade de quantificação do processo hidrológico. Os dados de ET são relevantes para o monitoramento ambiental, pois esses podem estar associados à perda de água vinculada à degradação da vegetação.

Dados de sensoriamento remoto por satélite têm o potencial para fornecer informações detalhadas sobre as propriedades da superfície da terra, mediante as imagens orbitais, que crescem como alternativa rápida de coletar informações sobre os fenômenos existentes na superfície da terra em nível local ou regional. Um dos parâmetros mais importantes provenientes da superfície da terra que podem ser derivados por meio dos sensores é evapotranspiração real. Assim, as imagens de satélite tornam-se ferramenta importante para o mapeamento e evolução temporal da evapotranspiração.

Nos últimos anos, têm sido propostos alguns algoritmos para estimativa dos componentes do balanço de energia à superfície, levando em consideração a variabilidade espacial de muitas variáveis micrometeorológicas. Bastiaanssen et al. (1998) propuseram um modelo semiempírico com abordagens intermediárias: o Surface Energy Balance Algorithm for Land (SEBAL). Esse algoritmo foi desenvolvido para estimativa dos componentes do balanço de energia (BE) e, por conseguinte, da evapotranspiração real, baseado em combinações de relações empíricas e parametrizações físicas (BASTIAANSSEN et al., 1998a,b). Utiliza apenas a radiância espectral registrada em sensores de satélites e um conjunto mínimo de dados meteorológicos de superfície, entre os quais a velocidade do vento e a temperatura do ar para solucionar o balanço radiativo e energético à superfície (COURALT et al., 2003).

Compaoréetal.(2008)afirmaram queoSEBAL destaca-se entre os demais algoritmos pelas seguintes razões: (a) consiste em um algoritmo baseado fisicamente em análises de imagens de satélite e requer um mínimo de informações meteorológicas; (b) faz uso de grande número de variáveis ambientais e não as assume constantes espacialmente como é feito em muitos outros métodos; (c) é reduzida a necessidade da correção atmosférica das informações em comprimentos de onda curta e térmica nas imagens, desde que a ET estimada dependa apenas de diferenças da temperatura radiométrica na cena em vez do valor absoluto da temperatura de superfície. Isso amplia a aplicabilidade do SEBAL, uma vez que as medições necessárias para correções atmosféricas não estão frequentemente disponíveis. O uso do SEBAL tem a grande vantagem de proporcionar o balanço de energia à superfície de maneira efetiva e econômica, possibilitando grande cobertura espacial e, dependendo do sensor orbital que o alimenta com dados dos canais reflectivos e termais, pode também ter grande resolução espacial (SILVA et al., 2005). 
Em estudos destinados à obtenção das componentes do balanço de energia são realizados com maior frequência nas medições de Rn (AZEVEDO et al., 2003, 2007; SILVA et al., 2007; ZHANG et al., 2008). Nesse sentido, Silva et al. (2005) obtiveram Rn em áreas do semiárido brasileiro, enquanto Di Pace et al. (2008) analisaram o impacto da topografia no cômputo do Rn. Já Gomes et al. (2009) avaliaram alterações nos balanços de radiação e energia decorrentes da substituição da vegetação natural por cultivos agrícolas.

A Área de Preservação Ambiental da Ilha de Santa Rita/Alagoas (APA Santa Rita) tem como objetivo preservar as características ambientais e naturais das regiões dos canais e lagoas "Mundaú” e "Manguaba”. Essa APA apresenta maior diversidade de cobertura vegetal, registrando-se a presença de restinga, manguezais, áreas de cultivo de coco e povoados com ocupação desordenada de bares, restaurantes e residências de veraneio. Ressalta-se, entretanto, que essa área vem passando por um processo de degradação acelerado, evidenciado pela existência do Polo Cloroquímico de Alagoas na área de tabuleiros, o que exigiu na época a abertura da Rodovia BR-424 e implantação de uma ponte sobre o canal de dentro, descaracterizando as áreas de manguezais.

Nesse contexto, este trabalho teve como objetivo analisar o comportamento espaço-temporal da evapotranspiração real diária na Área de Preservação Ilha de Santa Rita/Alagoas, tomando como subsídio as possíveis alterações no uso e cobertura do solo, a partir do uso do algoritmo SEBAL, imagens do satélite Landsat/TM-5 e dados complementares de estação meteorológica localizada na área de estudo.

\section{MATERIAL E MÉTODOS}

A área de estudo está localizada na Ilha de Santa Rita, no Estado de Alagoas. A Ilha de Santa Rita está localizada entre os Municípios de Maceió, Marechal Deodoro e Coqueiro Seco, limitando-se a região compreendida pelas seguintes coordenadas: latitudes $9^{\circ} 37^{\prime} 30^{\prime \prime}$ e $9^{\circ} 47^{\prime} 30^{\prime \prime}$ Sul e as longitudes $35^{\circ} 46^{\prime}$ 00” e 35 55'00', Oeste de Greenwich, Datum WGS84. Essa região foi denominada Área de Preservação Ambiental, pela Lei $n^{\circ} 4.607$, de 1984, e regulamentada pelo Decreto $n^{\circ}$ 6.274, de 5 de junho de 1985 (Área de Preservação Ambiental da Ilha de Santa Rita/Alagoas - APA Santa Rita).

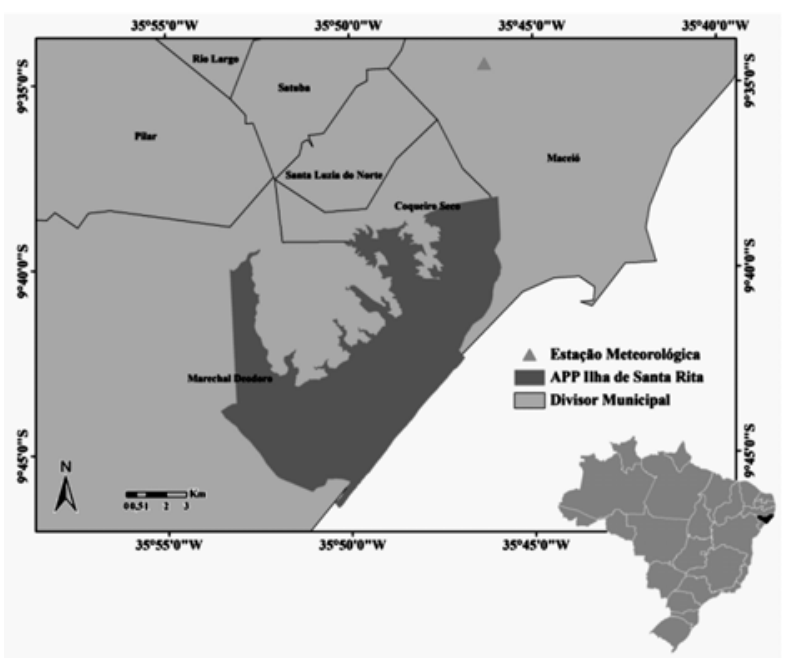

Figura 1 - Mapa de localização da área de estudo APA Santa Rita.

Figure 1 - Location map of the study area APA Santa Rita.

Na implementação do algoritmo SEBAL, foram utilizadas três imagens geradas pelo satélite Landsat/ TM-5, adquiridas na Divisão de Geração de Imagens (DGI) do Instituto Nacional de Pesquisas Espaciais (INPE), para órbita/ponto 214/067. As datas das imagens utilizadas são: três de novembro de 2003, 26 de outubro de 2006 e 11 de março de 2011. Os valores de velocidade do vento para a área de estudo foram adquiridos na Secretaria de Estado do Meio Ambiente e dos Recursos Hídricos de Alagoas (SEMARH/DMET). A estação meteorológica utilizada encontra-se localizada no Município de Maceió/Alagoas, com coordenadas: 9³3'03'”S, 3546'12'”W. A correção geométrica das imagens do Landsat 5 foi realizada no software ENVI, utilizando a imagem ortorretificada GeoCover 2000, disponibilizado em http://zulu.ssc.nasa.gov/mrsid/ mrsid.pl. O GeoCover 2000 são mosaicos ortorretificados gerados a partir de imagens Landsat 7/ ETM+, considerando as bandas 7, 4, 2 e 8, com resolução espacial de $14,5 \mathrm{~m}$. Foram utilizados 20 pontos de controle. A reamostragem dos pixels na imagem foi realizada pelo método do vizinho mais próximo, e o erro quadrático médio (RMS) ficou em torno de 1,2 pixel. Utilizaram-se o sistema projeção UTM e o elipsoide WGS-84.

O SEBAL é um método baseado fisicamente no processo analítico de imagens de satélite. Permite a determinação dos componentes do balanço de energia e da evapotranspiração real. Uma das primeiras etapas 
do SEBAL é a estimativa do saldo de radiação. As etapas percorridas para obtenção de Rn são mostradas na Figura 2. Os tópicos a seguir elucidam as etapas apresentadas no fluxograma da Figura 2.

Após o cálculo de Rn, partiu-se para a obtenção do fluxo de calor no solo (G), que pode ser definido como armazenamento de calor no solo e na vegetação devido à condução. O fluxo de calor do solo foi obtido de acordo com Bastiaanssen (2002):

$G=\left[\frac{T_{S}}{\alpha}\left(0,0038 \alpha+0,0074 \alpha^{2}\right)\left(0,98 N D V I^{4}\right)\right] R_{n}$

em que $\mathrm{T}_{\mathrm{s}}$ é a temperatura da superfície $\left({ }^{\circ} \mathrm{C}\right), 5 \varnothing^{\mathrm{a}} \beta$ é o albedo da superfície e NDVI é o índice de vegetação por diferença normalizada. Para efeitos de correção dos valores do fluxo de calor no solo para corpos de água (NDVI<0), Allen et al. (2002) sugeriram a utilização de $G=0,5 R n$.
A determinação do fluxo de calor Sensível (H) é a parametrização do SEBAL, que requer maior atenção, pois nesta etapa são introduzidas algumas hipóteses importantes. A despeito disso, tem-se a escolha dos pixels âncoras, a saber: pixel quente e frio. O SEBAL utiliza esses pixels para fixar as condições de contorno para o equilíbrio energético, os quais são localizados na área de interesse. O fluxo de calor sensível, segundo Bastiaanssen et al. (1998a), pode ser obtido pela expressão:

$$
H=\rho c_{p} \frac{a+b T_{s}}{r_{a h}}
$$

em que $\rho$ é a massa específica do ar, c é o calor específico do ar (1.004 $\left.\mathrm{Jkg}^{-1} \mathrm{k}^{-1}\right)$, a e b são constantes de calibração da equação linear estabelecida entre a diferença de temperatura em dois níveis $Z_{1}$ e $Z_{2}$ e a temperatura da superfície $\mathrm{T}_{\mathrm{S}}$ e $\mathrm{r}_{\mathrm{ah}}$ é a resistência aerodinâmica ao transporte de calor.

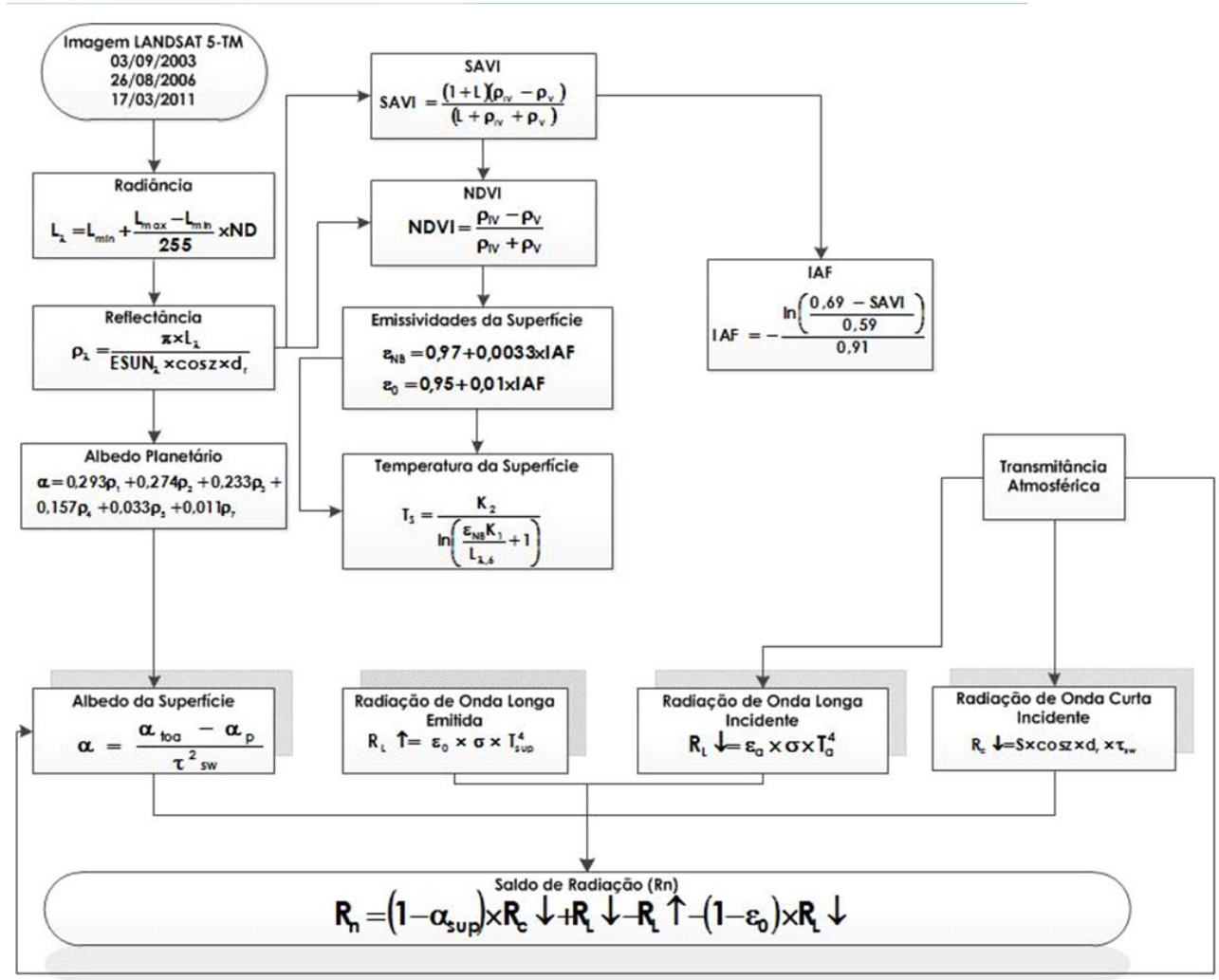

Figura 2 - Fluxograma de obtenção do saldo de radiação (Rn).

Figure 2 - Flowchart of radiation balance obtention (Rn).

Revista Árvore, Viçosa-MG, v.38, n.3, p.453-460, 2014 
Com o SEBAL, a Evapotranspiração Real é determinada com base na fração evaporativa instantânea - (FEi), definida como razão entre LE e Rn - G. Estudos confirmam que FEi é igual a FE (BASTIAANSEEN, 2000). Logo:

$$
F E_{i}=\left(\frac{L_{E}}{R_{n}-G}\right)=F E_{24 h}=\frac{L E_{24 h}}{R_{24 h}}
$$

Admite-se que $\mathrm{G}_{24 \mathrm{~h}}=0$. Assim, a $\mathrm{LE}_{24 \mathrm{~h}}$ é dada conforme a seguinte equação:

$$
L E_{24 h}=F E_{i} \times R_{24 h}
$$

em que $\mathrm{R}_{24 \mathrm{~h}}$ é o saldo de radiação diário e calculado conforme a equação 5 :

$$
R_{24 h}=R_{s, 24 h}(1-\text { albedo })-a \mathrm{~T}_{24 h}
$$

em que o coeficiente $a$ é, em média, de 143 para nossas condições semiáridas (TEXEIRA et al., 2008a). Segundo Teixeira et al. (2008a), esse valor é superior ao originalmente sugerido de 110 encontrado para a Holanda. A diferença pode ser atribuída a diferentes condições térmicas entre os macroclimas (TEIXEIRA et al., 2008b). Nesse sentido, a $\mathrm{ET}_{24 \mathrm{~h}}$ é dada por:

$$
L E T_{24 h}=F E_{\text {inst }} \times R_{24 h}
$$

\section{RESULTADOS E DISCUSSÃO}

As Figuras 3, 4 e 5 mostram a distribuição espacial da evapotranspiração real diária $\left(\mathrm{ET}_{24 \mathrm{~h}}\right)$. Os mapas de $\mathrm{ET}_{24 \mathrm{~h}}$ ilustram que os valores mais elevados foram encontrados sobre os corpos de água, Lagoas (Mundaú e Manguaba) e oceano Atlântico, alcançando valores próximos a 6 mm.dia-1. Bezerra (2008) encontrou para espelhos d'água na Chapada do Araripe valor semelhante. Acrescenta-se que Santos (2010), utilizando imagens ASTER, encontrou valores entre 6,41 e 7,81 mm.dia-1 enquanto Sun (2010), com imagens Landsat $7 \mathrm{ETM}^{+}$, obteve valores na faixa de 7 a 8 mm.dia ${ }^{-1}$.

As áreas em rosa, que apresentam $\mathrm{ET}_{24 \mathrm{~h}}$ na faixa de 0 a $1 \mathrm{~mm}$. dia $^{-1}$, foram observadas em regiões urbanizadas e em alguns pontos isolados de solo exposto para os dias 03/09/2003 e 26/08/2006. No dia 17/03/2011, em áreas urbanizadas, observou-se a elevação dos valores para a faixa de 1 a 2 mm.dia ${ }^{-1}$.

Mudança expressiva na evapotranspiração real sobre a vegetação nativa foi observado nos dias analisados. Em 03/09/2003, essa área ficou com valores na faixa de 2 a 3 mm.dia ${ }^{-1}$, em 26/08/2006, com valores entre 3 e 4 mm.dia $^{-1}$ e, em 17/03/2011, com valores superiores a 5 mm.dia ${ }^{-1}$. Tais valores se assemelham aos encontrados por Andrade (2012) na região do Pantanal, em que a $\mathrm{ET}_{\text {diária }}$ variou de 4,0 a 5,5 mm d ${ }^{1}$ em áreas com presença predominante de corpos d’água.

$\mathrm{Na}$ região de agricultura, os valores de $\mathrm{ET}_{24 \mathrm{~h}}$ sofreram variações bem perceptíveis entre os dias analisados. No dia 03/09/2003, quando a área agrícola é menor quando comparado com os demais dias analisados, os valores de $\mathrm{ET}_{24 \mathrm{~h}}$ em alguns pontos ficaram



$03 / 09 / 2003$

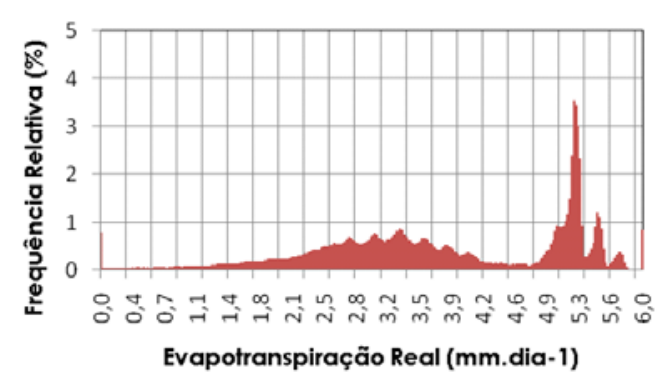

Figura 3 - Distribuição espacial da evapotranspiração real diária (mm.dia ${ }^{-1}$ ) e (b) histograma de frequência relativa, em 3 de setembro de 2003.

Figure 3 - Spatial distribution of real daily evapotranspiration $(\mathrm{mm}$.day-1) and $(\mathrm{b})$ relative frequency histogram on September 3rd, 2003.

Revista Árvore, Viçosa-MG, v.38, n.3, p.453-460, 2014 
na faixa de 2 a 3 mm.dia ${ }^{-1}$. Entretanto, no dia 17/ 03/2011, a região destinada à agricultura compreendeu valores de $\mathrm{ET}_{24 \mathrm{~h}}$ que variaram em diversas faixas, devido aos diferentes estágios de crescimentos da cana-deaçúcar. Foram encontrados $\mathrm{ET}_{24 \mathrm{~h}}<1 \mathrm{~mm} \cdot \mathrm{dia}^{-1}$ nas áreas de solo exposto.

Os valores de ET obtidos corroboram com os dados de Gomes (2009) que obteve valores inferiores a $1 \mathrm{~mm} \cdot$ dia $^{-1}$ correspondentes a áreas desprovidas de vegetação e 2 a 3 mm.dia ${ }^{-1}$. Santos (2010), com imagens ASTER, obteve valores inferiores a 3,96 mm.dia ${ }^{-1}$; Yang



$26 / 08 / 2006$



Figura 4 - Distribuição espacial da evapotranspiração real diária (mm.dia-1 ${ }^{-1}$ e histograma de frequência relativa em 26 de agosto de 2006.

Figure 4-Spatial distribution of real daily evapotranspiration (mm.day-1) and relative frequency histogram on August 26th, 2006.

Revista Árvore, Viçosa-MG, v.38, n.3, p.453-460, 2014
(2012), a partir de dados do sensor MODIS, em regiões de vegetação esparsa ou nua, conseguiu valores inferiores a 2,5 mm.dia ${ }^{-1}$; e Sun (2010), no solo exposto, valores na faixa de 0 a $1 \mathrm{~mm} \cdot \mathrm{dia}^{-1}$. Esses dados, de maneira geral, fortalecem as estimativas de ET obtidas neste trabalho.

Um grande fator determinante para a estimativa da ET pelo SEBAL é a temperatura de superfície. Em regiões desprovidas de cobertura vegetal são encontrados valores elevados de temperatura de superfície e redução nos valores de ET. Isso pode ser observado

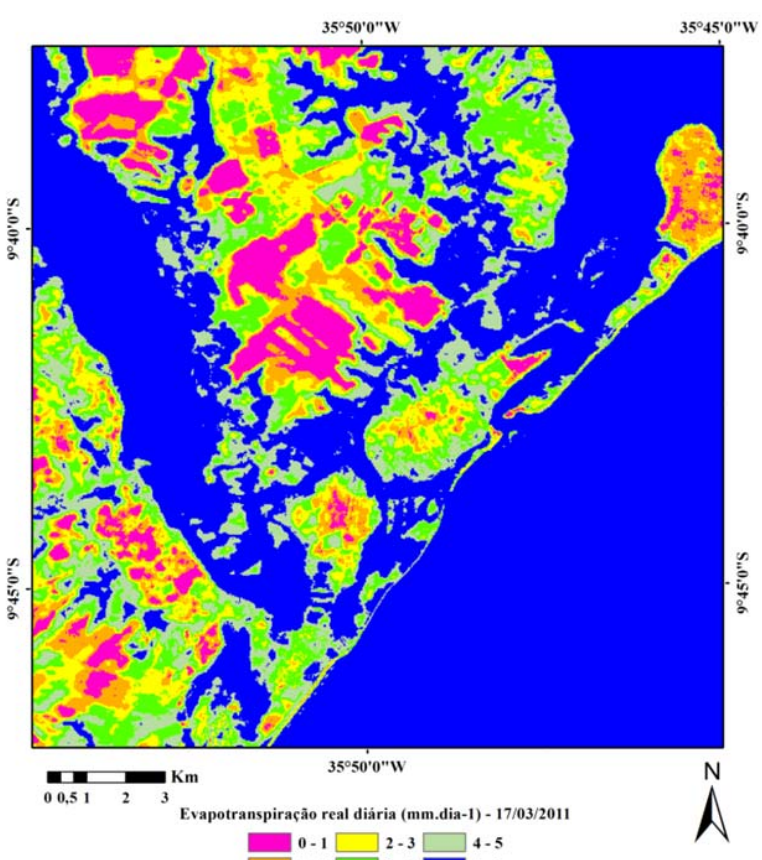

$17 / 03 / 2011$

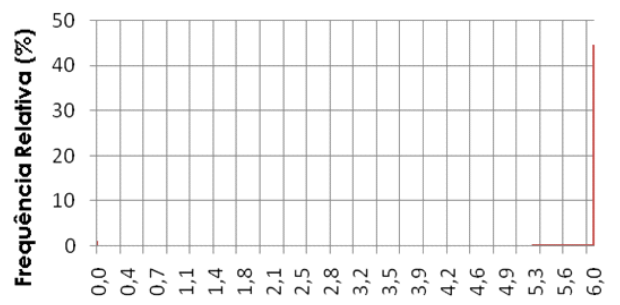

Evapotranspiração Real (mm.dia' $\left.{ }^{-1}\right)$

Figura 5 - Distribuição espacial da evapotranspiração real diária $\left(\mathrm{mm}_{\text {.dia }}{ }^{-1}\right)$ e histograma de frequência relativa em 17 de março de 2011.

Figure 5 - Spatial distribution of real daily evapotranspiration (mm.day-1) and relative frequency histogram on March 17th, 2011. 
em alguns trabalhos, como em Bezerra (2008) com Landsat, Santos (2010) com ASTER, Sun (2010) com Landsat 7/ $\mathrm{ETM}^{+}$e Yang (2012) com o MODIS.

Os dados são indicativos de que as mudanças ocorridas na APA Santa Rita verificadas na temperatura da superfície, no saldo e na evapotranspiração, foram influenciadas em áreas antes provenientes de vegetação natural no período de 2003 a 2011, pela implantação do condomínio Laguna. Precisamente para essa região, foram evidenciados diminuição de ET e aumento da temperatura de superfície. Em 2003, a faixa de ET variou entre 3 e $4 \mathrm{~mm}$ dia $^{-1}$, e sua temperatura encontrava-se em 294 ${ }^{\circ} \mathrm{C}$. Em 2011, a ET apresentou valores inferiores a $1 \mathrm{~mm}$.dia ${ }^{-1}$, com temperatura de superfície de $306{ }^{\circ} \mathrm{C}$. Logo, tais valores podem ser justificados pelo desmatamento ocasionado nessa região.

Diante dos aspectos apresentados, fica evidenciado que a descaracterização natural de uma região pode ocasionar a elevação dos valores atmosféricos.

\section{CONCLUSÕES}

Neste estudo, a aplicação da técnica do SEBAL foi conduzida para analisar o comportamento espaço temporal da ET, tomando como subsídio as possíveis alterações de uso e cobertura do solo. Os valores estimados de ET foram comparados entre si, nos dias analisados, sendo consideradas as possíveis alterações antrópicas, na região. Esse fato pode ser evidenciado pela presença de um condomínio residencial na APA. Diante dos resultados e da discussão, a seguir são apresentadas as seguintes conclusões:

A estimativa diária de $\mathrm{ET}_{24 \mathrm{~h}}$ real variou de $0 \mathrm{a}$ $1 \mathrm{~mm}$. dia $^{-1}$ em solo exposto e área urbanizada, 6 mm.dia ${ }^{-1}$ em corpos d'água e mangue. $\mathrm{ET}_{24 \mathrm{~h}}$ real apresentou valores médios de 3,78 mm.dia ${ }^{-1}$, 3,45 mm,dia e 4,75 mm.dia- $^{-1}$ ${ }^{1}$, nos dias 03/09/2003, 26/08/2006 e 17/03/2011, respectivamente, em toda a área estudada.

- A variação da $\mathrm{ET}_{24 \mathrm{~h}}$ real estimada em diferentes tipos de uso e cobertura do solo sugere a aplicação da abordagem SEBAL, com algumas informações detalhadas, a exemplo do campo de cultura. No entanto, observou-se que a evapotranspiração é distinta em diferentes estágios de fenológicos.

- Diante da redução de áreas de vegetação nativa, observou-se redução nos valores evaporativos.
- Os recursos de compartilhamento de energia estão concentrados nas áreas rurais e urbanas. Nas áreas de menos cobertura vegetal, como áreas industriais e comerciais, observou-se a elevação da temperatura da superfície.

- Os resultados obtidos por meio do algoritmo SEBAL podem ser utilizados em condições climáticas semelhantes às da Área de Preservação Ilha de Santa Rita/Alagoas. As distribuições de cada componente - saldo de radiação, fluxo de calor no solo, sensível e latente - têm influência significativa nos diferentes tipos de uso e cobertura do solo. Além disso, existem diferenças evidentes entre os fluxos de calor em zona rural e área urbana.

- A abordagem utilizada é muito adequada para uma exploração real de dados de satélite, sendo recomendada para estimar um número de parâmetros no contínuo solo-planta-atmosfera. Esses parâmetros têm a vantagem de ser espacializados e proporcionar uma cobertura espaçotemporal melhor do que os pontos de dados medidos operacionalmente.

\section{AGRADECIMENTOS}

Ao Programa de Pós-Graduação em Ciências Geodésicas e Tecnologias da Geoinformação-DECART/ UFPE e à Secretária de Estado do Meio Ambiente e Recursos Hídricos de Alagoas, pelo apoio à realização da pesquisa.

\section{REFERÊNCIAS}

ANDRADE, R. G. et al. Geotecnologias aplicadas à avaliação de parâmetros biofísicos do Pantanal. Pesquisa Agropecuária Brasileira, v.47, n.9, p.1227-1234, 2012.

ALLEN, R. G. Using the FAO-56 dual crop coefficient method over an irrigated region as part of an evapotranspiration intercomparison study. Journal of Hydrology, v.229, n.1, p.27-41, 1998.

ALLEN, R. G. Metal. Surface Energy Balance Algorithm for Land (SEBAL) - Advanced training and users; manual. Idaho, 2002. 98p.

ALLEN, P.L.; HOWELL, T.; JENSEN, M. Evapotranspiration information reporting: Factors governing measurement. Agricultural Water Management, v 98, n.6, p.899-920, 2011.

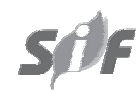

Revista Árvore, Viçosa-MG, v.38, n.3, p.453-460, 2014 
AZEVEDO, P. V.; SILVA, B. B.; SILVA, V. P. $\mathrm{R}$.Water requirements of irrigated mango orchards in northeast Brazil. Agricultural Water Management, v.58, n.3, p.241-254, 2003.

AZEVEDO, P. V. et al.. Water requirements of pineapple crop grown in a tropical environment. Agricultural Water Management, v.88, n.1-3, p.201-208, 2007.

BASTIAANSSEN, W. G. M. SEBAL-based sensible and latent heat flux in the irrigated Gediz basin, Turkey Journal of Hydrology, v.229, n.1, p.87-100, 2000.

BASTIAANSSEN, W. G. M.; AHMAD, M-UD-Din; CHEMIN, Y. Satellite surveillance of evaporative depletion across the Indus Basin. Water Resources Research, v.38, n.12 (disponível em http://onlinelibrary.wiley.com/doi/10.1029/ 2001WR000386/full), 6 dec 2002.

BASTIAANSSEN, W. G. M. et al. A remote sensing surface energy balance algorithm for land (SEBAL): 2. Validation. Journal of Hydrology, v.212/213, p.213-229, 1998.

BEZERRA, B. G. et al. Estimativa da evapotranspiração real diária utilizando-se imagens digitais TM - LANDSAT 5. Revista Brasileira de Meteorologia, v.23, n.3, 305317, 2008.

COMPAORÉ, H. et al. Evaporation mapping at two scales using optical imagery in the White Volta Basin, Upper East Ghana. Physics and Chemistry of the Earth, v.33, n.1, p.127140, 2008.

COURALT, D.; SEGUIM, B.; OLIOSO, A. "Review To Estimate Evapotranspiration From Remote Sensing Data: Some Examples From The Simplified Relationship To The Use Of Mesoscale Atmospheric Models." ICID Workshop On Remote Sensing Of ET For Large Regions, 17th September, 2003.

DI PACE, F. T. et al. Mapeamento do saldo de radiação com imagens Landsat 5 e modelo de elevação digital. Revista Brasileira de Engenharia Agrícola e Ambiental, v. 12, n.4, p.385-392, 2008.
GOMES, H. B. Balanços de radiação e energia em áreas de cultivo de canade -açúcar e cerrado no estado de São Paulo mediante imagens orbitais. 2009. 108f. Tese (Doutorado em Meteorologia) Universidade Federal de Campina Grande, Campina Grande, 2009.

GOMES, H. B. et al. Balanço de radiação em diferentes biomas no Estado de São Paulo mediante imagens Landsat 5. Geociências, v.28, n.1, p.153-164, 2009.

IRMAK, A. Evapotranspiration - Remote sensing and modeling. Published by InTech, DOI: 10.5772/725 , Croatia. Dec., 2011.

SANTOS, T. V. et al. Avaliação de fluxos de calor e evapotranspiração pelo modelo SEBAL com uso de dados do sensor ASTER. Pesquisa

Agropecúaria Brasileira, v.45, n.5, p.488496, 2010.

SILVA, B. B.; LOPES, G. M.; AZEVEDO, P. V. Determinação do albedo de áreas irrigadas com base em imagens Landsat 5 - TM. Revista Brasileira de Agrometeorologia, v.13, n.2, p.11-21, 2005.

SILVA, V. P. R.; AZEVEDO, P. V.; SILVA B. B. Surface energy fluxes and evapotranspiration of a mango orchard grown in a semiarid environment.

Agronomy Journal, v.99, n.6, p.1391-1396, 2007.

TEIXEIRA, A. H. C. et al. Reviewing SEBAL input parameters for assessing evapotranspiration and water productivity for the Low-Middle São Francisco River basin, Brazil Part B: Application to the regional scale in Pernambuco.

Agricultural and Forest Meteorology, v.149, p.462-476, 2009.

YANG, Y. et al. Remote sensing temporal and spatial patterns of evapotranspiration and the responses to water management in a large irrigation district of North China. Agricultural and Forest Meteorology, n.164, p.112-122, 22, 2012.

ZHANG, B. et al. Comparison of three evapotranspiration models to Bowen ratio-energy balance method for a vineyard in an arid desert region of northwest China. Agricultural and Forest Meteorology, v.148, n.10, p.1629-1640, 2008. 